\title{
BIOPSYCHOSOCIAL DETERMINANTS OF TERTIARY PREVENTIVE BEHAVIORS AMONG PATIENTS WITH HYPERTENSION IN SRAGEN, CENTRAL JAVA
}

\author{
Very Retnowati'), Pawito's), Bhisma Murti') \\ 1)Masters Program in Public Health, Universitas Sebelas Maret \\ 2)Faculty of Social and Political Science, Universitas Sebelas Maret
}

\begin{abstract}
Background: Tertiary hypertension prevention is an effort to prevent hypertensive patients from disabilities and complications, which lead to improve their quality of life. The biopsychosocial aspect influences individual behavior in maintaining blood pressure. This study aimed to analyze the biopsychosocial determinants of tertiary prevention behavior in hypertensive patients.

Subjects and Method: A cross sectional study was conducted at 25 integrated health posts (posbindu) in Sragen, Central Java. A sample of 200 hypertensive patients was selected by stratified random sampling. The dependent variable was tertiary hypertension preventive behavior. The independent variables were attitude, observational learning, role model, imitation, vicarious learning, reinforcement, self-efficacy, self-regulation, and outcome expectation. The data were collected by questionnaire and analyzed by a multiple logistic regression.

Results: Tertiary hypertension preventive behavior increased with observational learning $(\mathrm{b}=1.83 ; 95 \% \mathrm{CI}=0.31$ to $3.35 ; \mathrm{p}=0.018)$, role model $(\mathrm{b}=1.95 ; 95 \% \mathrm{CI}=0.75$ to $3.16 ; \mathrm{p}=$ o.001), imitation $(\mathrm{b}=2.13 ; 95 \% \mathrm{CI}=0.89$ to $3.38 ; \mathrm{p}=0.001)$, vicarious learning $(\mathrm{b}=1.60$; $95 \% \mathrm{CI}=0.23$ to $2.96 ; \mathrm{p}=0.022)$, reinforcement $(\mathrm{b}=2.86 ; 95 \% \mathrm{CI}=1.25$ to $4.47 ; \mathrm{p}<0.001)$, self-efficacy $(b=1.99 ; 95 \% \mathrm{CI}=0.73$ to $3.25 ; \mathrm{p}=0.002)$, self-regulation $(\mathrm{b}=1.39 ; 95 \% \mathrm{CI}=$ 0.18 to $2.61 ; \mathrm{p}=0.024)$, outcome expectation $(\mathrm{b}=2.37 ; 95 \% \mathrm{CI}=0.85$ to $3.89 ; \mathrm{p}=0.002)$, and positive attitude $(\mathrm{b}=1.76 ; 95 \% \mathrm{CI}=0.40$ to $3.13 ; \mathrm{p}=0.011)$.

Conclusion: Tertiary hypertension preventive behavior increases with observational learning, role model, imitation, vicarious learning, reinforcement, self-efficacy, selfregulation, outcome expectation, and positive attitude.
\end{abstract}

Keywords: hypertension, tertiary prevention, biopsychosocial, social cognitive theory

\section{Correspondence:}

Very Retnowati. Masters Program in Public Health, Universitas Sebelas Maret. Jl. Ir. Sutami 36A, Surakarta 57126, Central Java. Email: veryretnowati@gmail.com. Mobile: $+6281548592491$

The $7^{\text {th }}$ International Conference on Public Health Solo, Indonesia, November 18-19, 2020 | 171 https://doi.org/10.26911/the7thicph.02.60 